\title{
Formation of Quark Phases in compact stars and their connection to Gamma-Ray-Bursts
}

\author{
A. Drago ${ }^{a}$, G. Pagliara ${ }^{b}$, J. Schaffner-Bielich ${ }^{b}$ \\ ${ }^{a}$ Dipartimento di Fisica - Università di Ferrara and INFN Sez. di Ferrara, 44100 \\ Ferrara, Italy \\ ${ }^{b}$ Institut für Theoretische Physik, Goethe Universität, D-60438, Frankfurt am Main, \\ Germany
}

\begin{abstract}
We analyse the occurrence of quiescent times in the temporal structure of the Gamma-Ray-Bursts (GRBs) light curves. We show that if a long quiescent time is present, it is possible to divide the total duration of GRBs into three periods: the pre-quiescence emission, the quiescent time and the post-quiescence emission. We then discuss a model of the GRBs inner engine based on the formation of quark phases during the life of an hadronic star. Within this model the pre-quiescence emission is interpreted as due to the deconfinement of quark inside an hadronic star and the formation of $2 \mathrm{SC}$ quark matter. The post-quiescence emission is due to the conversion of $2 \mathrm{SC}$ into the Color-Flavor-Locking (CFL) phase. The temporal delay between these two processes is connected with the nucleation time of the CFL phase in the 2SC phase and it can be associated with the observed quiescent times in the GRBs light curves. The stability of CFL cores in compact stars is also discussed.
\end{abstract}

\section{Introduction}

The time structure of GRBs is usually complex and it often displays, during the phase of the prompt emission, several short pulses separated by time intervals lasting from fractions of second to several tens of seconds. In some cases very long period of vanishing signal, the so called quiescent times (QTs) are present, which can have durations comparable with the durations of the emission periods. Within the internal shock model QTs shorter than few tens of seconds can be explained by the modulation of a continuous shells emission from the Inner Engine (IE) [1. When a long QT is present in the light curve, as we will show, it is more plausible to assume that the inner engine is dormant. We discuss then the quark deconfinement model of the GRBs. In this model the interpretation of long QTs is given in terms of time intervals between readjustments of the structure of a compact star. We suggest that the GRB emissions are due to first order phase transitions occurring between different phases of the strong interacting matter and we associate the periods of dormancy with the nucleation times needed to trigger the phase transitions. 
Formation of Quark Phases in compact stars and their connection to Gamma-Ray-Bursts2

\section{Quiescent times in the GRBs prompt emission}

A previous statistical analysis [2] has shown that there are three time-scales in the GRB light curves: the shortest one is the variability scale determining the pulses durations and the intervals between pulses; the largest one describes the total duration of the bursts and, finally, an intermediate time scale is associated with long periods within the bursts having no activity, the QTs. The origin of these periods of quiescence is still unclear. We have recently 3] performed a new statistical analysis of the time intervals $\Delta t$ between adjacent peaks in the light curve of GRBs using the algorithm introduced in Ref. [2]. We have applied this analysis to all the light curves of the BATSE catalogue. In a first investigation we have merged all the bursts of the catalogue into one sample from which we compute the cumulative probability $c(\Delta t)$ of finding time intervals $\Delta t$ which are not QTs i.e. we compute the distribution of the time intervals within each active period. In Fig. 1a, we show that $c(\Delta t)$ is well described by a log-normal distribution. In Fig. 1b, the histogram of QTs is displayed together with a log-normal distribution. As already observed by previous authors [2], there is an evident deviation of the data points respect to the log-normal distribution for time intervals longer than a few seconds, indicating an excess of long $\Delta t$. In Fig. 10 we show a power law fit of the tail of the QTs distribution which displays a very good agreement with the data, as already observed by [4]. Finally, in Fig. 1d we show a correlation function, indicating the probability of finding at least 2 QTs longer than $\Delta T$ in a same GRB.This probability rapidly decreases and it essentially vanishes for $\Delta T>40 \mathrm{~s}$.

We can now define a subsample of the BATSE catalogue composed of all the bursts having a QT longer than $40 \mathrm{~s}$ and study its properties. From the result of Fig. 11d, the bursts of the subsample contain only one long QT and it is therefore possible to divide each burst into a pre-quiescence emission (PreQE) and a post-quiescence emission (PostQE) of which we will compare the temporal and spectral structure.

In Fig. 2 we display the cumulative distributions $c_{1}(\Delta t)$ and $c_{2}(\Delta t)$ within each of the two emission periods. The two distributions are very similar as also confirmed by the $\chi^{2}$-test. Let us remind that within the internal-external-shocks model [5, 6], external shocks produce emissions lacking the short time scale variability produced by internal shocks [7]. The result of Fig. 2 rules out a scenario in which PostQE is dominated by external shocks and PreQE by internal shocks. This in turn excludes the possibility of associating the QTs with the time needed for the jet to reach and interact with the interstellar medium.

The statistical analysis of the durations $D 1$ and $D 2$ of the two emission periods (see Ref. [3]) show that the two data sets are well fitted by two log-normal distributions. The two distributions have different mean values $\left(D 1_{\text {ave }} \sim 21 s, D 2_{\text {ave }} \sim 41 s\right)$ and almost identical standard deviations $\left(\sigma_{1}=36 s, \sigma_{2}=33 s\right)$. To estimate the emitted energy during PreQE and PostQE we have analysed the hardness ratios and the power emitted in each emission. Both quantities are on average the same. Let us now discuss the implications of this analysis on the origin of QTs. As observed by [1], within the 
Formation of Quark Phases in compact stars and their connection to Gamma-Ray-Bursts3
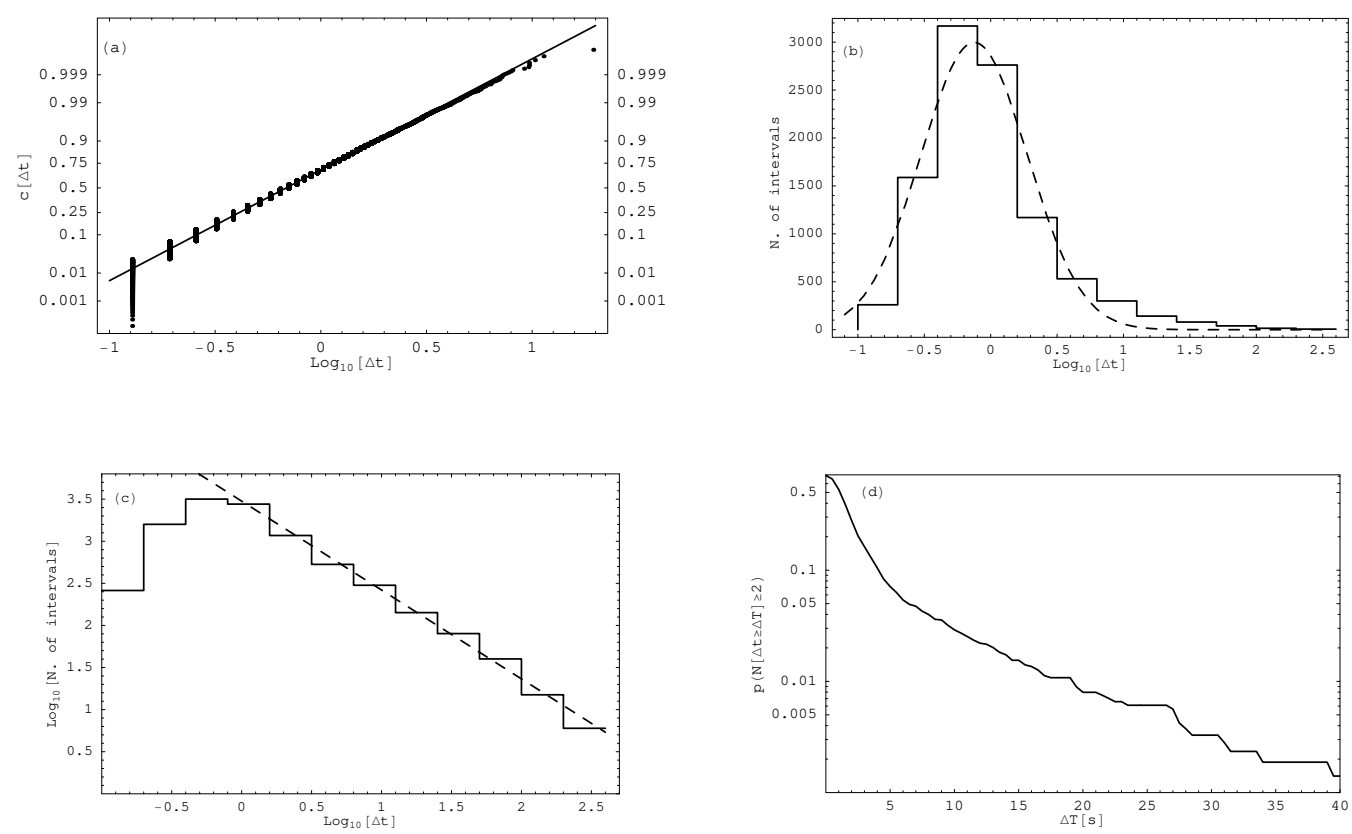

Figure 1. Analysis of time intervals between peaks a The cumulative distribution of time intervals $\Delta t$ which are not QTs (black point), is compared with its best fit log-normal distribution (solid line). b Histogram of the QTs and its log-normal fit (dashed line). c Histogram of QTs and power-low fit of its tail (dashed line). d Frequency of bursts containing at least two QTs longer than $\Delta T$.

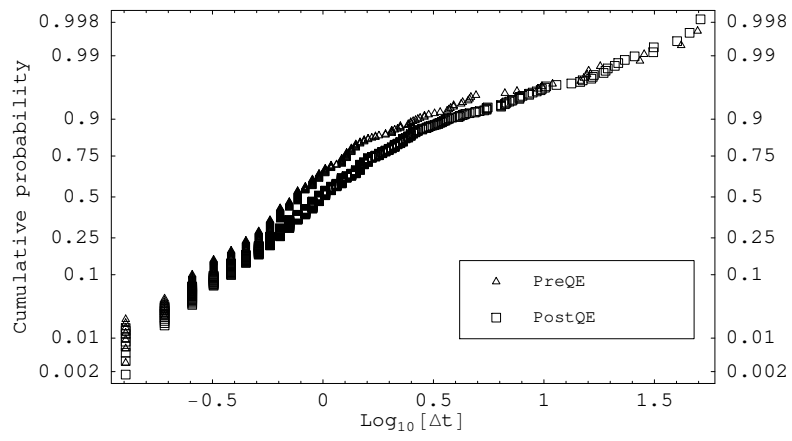

Figure 2. Analysis of time intervals between peaks within the two emission periods The cumulative distributions of $\Delta t$ are shown for the two emission episodes.

internal shocks model it is possible to explain the QTs either as a turn-off of the IE or as a modulation of a continuous relativistic wind emitted by the IE (Wind Modulation Model WMM). Both hypothesis are consistent with the result of Fig. 2, The main difference between the WMM and the dormant engine scenario is that in the WMM the inner engine has to provide a constant power during the whole duration of the burst. In our subsample, we have several bursts whose total duration (including the QT) approaches $300 \mathrm{~s}$. These durations have to be corrected taking into account the average redshift of the BATSE catalogue, $z_{\text {ave }} \sim 2$, but even after this renormalization, durations of a hundred seconds or more are not too rare. This time scale has to be compared with 
the typical duration of the emission period of the inner engine, as estimated in various models. For instance, in all numerical investigations of the collapsar model [8] the IE remains switched-on during some 20s. Also in the quark deconfinement model which will be discussed in the next section the inner engine remains active during periods of the order of a few ten seconds corresponding to the cooling time of the compact stellar object. We conclude therefore that the energy requirement within the WMM scenario is too large with respect to the results of the theoretical models. It is instead more plausible to assume that during a QT the inner engine switches off, sometimes for very long periods, and then restart producing a PostQE very similar to the PreQE.

\section{Phase transitions between QCD phases and CFL core in compact stars}

Let us now discuss how to generate dormancy periods in the prompt emission within the quark deconfinement model. In this model the energy source powering the GRB is the transition from a star containing only hadrons to a star composed, at least in part, of deconfined quarks [9]. In the first calculations within the quark deconfinement model, the equation of state (EoS) of quarks was computed using the MIT bag model. Actually, in the last years the possibility of forming a diquark condensate at the center of a compact star has been widely discussed in the literature [10]. It was shown that the formation of a color superconducting quark core can increase the energy released by a significant amount [11]. In particular, many calculations indicate that Color-FlavorLocked (CFL) quark matter is the most stable configuration at large density whereas at intermediate density the two flavor color superconducting $2 \mathrm{SC}$ phase, or the normal quark (NQ) phase depending on the parameters, are favoured [12, 13]. The transition from the 2SC matter to CFL matter can take place as a first order phase transition if the leptonic content of the newly formed normal quark matter phase is not too small [14]. It is therefore tempting to associate the PreQE with the transition from the hadronic to the 2SC phase and the PostQE with the formation of the CFL phase [15]. In this scenario the two dimensional scales regulating the durations of PreQE and PostQE are the energies released in the two transitions. Concerning the conversion process between different QCD phases, it turns out that the conversion always takes place as a strong deflagration and never as a detonation [16]. This is important because in the case of a detonation the region in which the electron-photon plasma forms (e.g. via neutrinoantineutrino annihilation near the surface of the compact star) would be contaminated by the baryonic load and it would be impossible to accelerate the plasma up to the enormous Lorentz factors needed to explain the GRBs.

We want to discuss now the possible formation of a CFL phase core inside compact stars. While it has been shown in MIT-inspired quark models that the CFL phase can appear in hybrid stars or purely quark stars [17, 15], up to now, this is not the case within NJL-inspired models: in hybrid stars the appearance of a CFL core renders the star unstable [18, 19]. Actually, in very recent study of the quark core of proto-neutron stars with the NJL model it has been show that in a tiny window of baryonic masses and 
for vanishing temperature and neutrino chemical potential a CFL core can form [20]. Nevertheless the conclusion of that work is that by taking into account the influence of the hadronic crust, the hybrid stars become unstable again when the central density reaches the onset of the CFL phase transition. As in Ref. [20], we want to discuss the possible formation of a CFL core in a proto-neutron star limiting the discussion to the EoS of the quark phase. A complete study including also the hadronic phase and the Gibbs construction, as made in Ref. [21, for the phase transition is still in progress. To compute the EoS of quark matter we use the NJL-like model of Ref.[12, 14]. The only difference with respect to the model discussed in Ref. [20] is the inclusion of the six-fermion interaction term in the Lagrangian which simulates the breaking of axial symmetry. As already observed in Ref. [18], this term is responsible for the flavormixing of quarks which in turn leads to a different behaviour of the strange quark mass $m_{s}$ as a function of the chemical potential : in the absence of the six-fermions interaction (i.e. choosing $K=0), m_{s}$ has the same value both in the chiral broken phase and in the intermediate 2SC (NQ) phase and then rapidly decreases at the onset of the CFL phase [19]. In the case of $K \neq 0, m_{s}$ jumps to lower values already in the 2SC (NQ) phase and then decreases again in the CFL phase [12]. This different behaviour has noticeable effects on the EoS. As already observed by Buballa [18, for $K \neq 0$ the phase transition from the $2 \mathrm{SC}$ to the CFL phase occurs at lower density. Moreover, the gap of the energy density at the onset of the 2SC-CFL transition is considerably lower when the six-fermion term is included as we can observe in the left panel of Fig. 3 where the EoS of quark matter is shown for a temperature of $30 \mathrm{MeV}$ and vanishing neutrino chemical potential $\mu_{\nu}$. This feature has interesting effects on the corresponding massradius relation of compact stars. In the right panel of Fig. 3, we show the mass-radius relations using the EoS discussed before for the cases $K \neq 0$ and $K=0$, with $T=30$ $\mathrm{MeV}, \mu_{\nu}=0$. CFL cores do exist for $K \neq 0$ in a sizeable window of masses. This is a consequence of the weaker phase transition between the 2SC and the CFL phases when the six-fermion interaction is included. It also interesting to observe that the CFL core can form already at large temperatures while in the case $K=0$ it can form only at vanishing temperatures. This could have important consequences for the evolution of the proto-neutron star. Computing the differences of gravitational masses between a 2SC and a CFL star having the same baryonic mass, values of few per cent of solar mass can be obtained which correspond to energies released in the conversion of the order of $10^{52}$ ergs. In our model this process powers the PostQE emission.

\section{Conclusions}

We have provided hints for the interpretation of long QTs in GRB as due to dormancy periods of the inner engine. Before and after a long quiescent time the temporal microstructures of the emissions are similar. Interestingly, the average duration of PostQE is longer than the average duration of PreQE indicating that probably a larger amount of energy is released by the inner engine in the PostQE. Within the quark 
Formation of Quark Phases in compact stars and their connection to Gamma-Ray-Bursts6
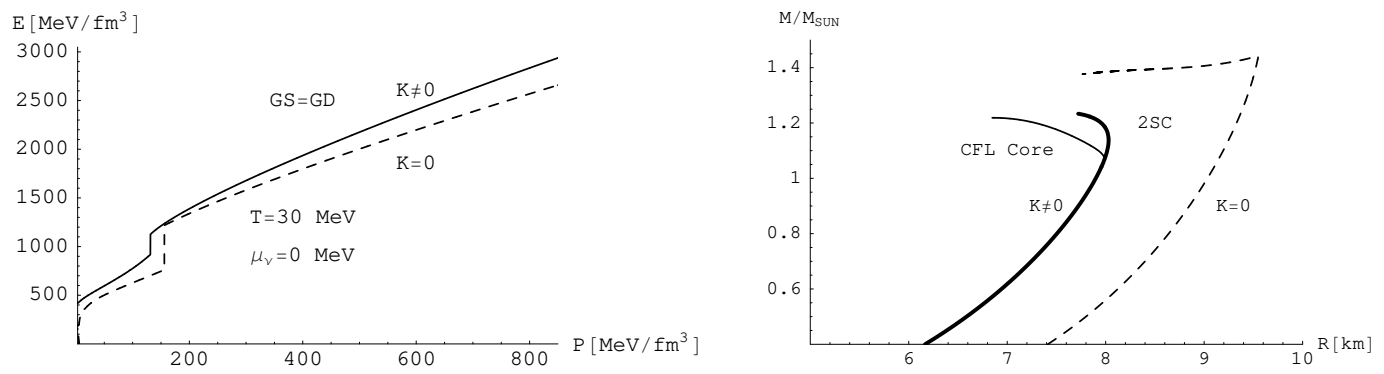

Figure 3. Equation of state and mass-radius relation Left panel:The energy density as a function of the pressure is shown for the two cases with and without the six-fermions interaction term $(K \neq 0, K=0)$ for $\mathrm{T}=30 \mathrm{MeV}$ and $\mu_{\nu}=0$. The diquark coupling constant is chosen to be equal the the quark-antiquark coupling constant (see [12, 14). Right panel: mass-radius relation for quark cores. The thin and thick lines correspond to the EoS with $K \neq 0$ with a transition from 2SC to the CFL phase and for only the 2SC phase respectively, the dashed line stands for the case $K=0$. When the CFL transition is reached in the first case a stable CFL core is possible; instead in the second case this leads to an instability of the star.

deconfinement model is possible to associate the PreQE with the deconfinement phase transition in which the 2SC phase is formed and the PostQE with the formation of the CFL phase. It has also been shown, considering only the quark EoS, that stable CFL cores can be formed in compact stars. On this point a more refined calculation is needed matching the quark EoS at large density with the hadronic EoS at low density.

\section{Acknowledgements:}

We thank S. Rüster and D. Rischke for providing us the code to compute the equation of state of quark matter. G.P. thanks INFN for financial support.

[1] Ramirez-Ruiz, E., Merloni, A., Rees, M.J., Mon. Not. Roy. Astron. Soc., 324, 1147 (2001).

[2] Nakar, E., Piran, T., Mon. Not. Roy. Astron. Soc., 331, 40 (2002).

[3] Drago, A, Pagliara, G., astro-ph/0512602 in press on Astrophys.J..

[4] Quilligan, F. et al., Astron. \& Astrophys., 385, 377 (2002).

[5] Piran, T., Rev.Mod.Phys., 76, 1143 (2004).

[6] Zhang, B., Meszaros, P., Int.J.Mod.Phys., A19, 2385 (2004).

[7] Sari, R., Piran, T., Astrophys.J., 485, 270 (1997).

[8] MacFadyen, A.L., Woosley, S.E., Astrophys.J., 524, 262 (1999).

[9] Berezhiani, Z., Bombaci, I., Drago, A., Frontera, F., Lavagno, A., Astrophys.J., 586, 1250 (2003).

[10] Rajagopal, K., Wilczek, F., hep-ph/0011333 (2000).

[11] Drago, A., Lavagno, A., Pagliara, G., Phys.Rev., D69, 057505 (2004).

[12] Ruster, S.B., Werth, V., Buballa, M., Shovkovy, I.A., Rischke D.H., Phys.Rev.D72: 034004 (2005).

[13] Blaschke, D., Fredriksson, S., Grigorian, H., Oztas, A.M., Sandin, F., Phys.Rev.D72:065020 (2005).

[14] Ruster, S.B., Werth, V., Buballa, M., Shovkovy, I.A., Rischke, D.H., Phys.Rev.D73: 034025 (2006).

[15] Drago, A., Lavagno, A., Pagliara, G., astro-ph/0510018, Proceedings QM2005.

[16] A. Drago, A. Lavagno, I. Parenti, astro-ph/0512652 in press on Astrophys.J.

[17] M. Alford and S. Reddy, Phys.Rev.D67 074024 (2003).

[18] M. Buballa, Phys.Rept.407 205 (2005).

[19] T. Klahn et al. nucl-th/0609067.

[20] F. Sandin, D. Blaschke, astro-ph/0701772

[21] Schertler, Leupold K.S., Schaffner-Bielich, J., Phys.Rev.C60:025801 (1999). 